\title{
Gravity in a lattice Boltzmann model
}

\author{
J. M. Buick and C. A. Greated \\ Department of Physics and Astronomy, The University of Edinburgh, The Kings Buildings, Mayfield Road, \\ Edinburgh, EH9 3JZ, United Kingdom
}

(Received 26 March 1999; revised manuscript received 19 November 1999)

\begin{abstract}
In this paper we consider the introduction of a body force, in the incompressible limit, into the lattice Boltzmann model. A number of methods are considered and their suitability to our objectives determined. When there is no density variation across the fluid, gravity can be introduced in the form of an altered pressure gradient. This method correctly satisfies the Navier-Stokes equation; however, if there is a non-negligible density variation present (produced by the body force or otherwise) this method becomes less accurate as the density variation increases and the constant density approximation becomes less valid. Three other methods are also considered for application when there is a non-negligible density variation. The equations of motion satisfied by these models are found up to second order in the Knudsen number and it is seen that only one of these methods satisfies the true Navier-Stokes equation. Numerical simulations are performed to compare the different models and to assess the range of application of each.
\end{abstract}

PACS number(s): 47.11.+j, 02.70.-c

\section{INTRODUCTION}

A recent development in the computational study of fluids has been the lattice Boltzmann model [1-4] which has developed from the lattice-gas automata [5]. This has been used successfully to simulate many problems including magnetohydrodynamics [6], turbulence $[7,8]$ colloidal suspensions [9], and multiphase flow [10-12]. Lattice Boltzmann simulations have traditionally been performed on a regular grid, however, it has recently been shown that, with the inclusion of an interpolation step, the technique can be applied on an irregular grid with the introduction of only a small error [13].

There is a wide range of fluid problems in which gravity and buoyancy effects are significant, for example, the study of water waves [14-16]. In this paper we consider the inclusion of a body force in the lattice Boltzmann scheme. We begin by describing the lattice Boltzmann model and showing that the model does indeed mimic the Navier-Stokes equation. Different methods for implementing gravity into the model are then considered and their ability to satisfy the Navier-Stokes equation is assessed. A number of simulations involving gravity are presented to verify the theoretical conclusions.

Here we are concerned with simulating gravity in the incompressible limit of a linearly varying density. In this limit we require $g z \ll c_{s}^{2}$, where $g$ is the gravitational strength, $z$ is the vertical extent of the simulation, and $c_{s}$ is the speed of sound. In this limit $g$ can have a significant value so it is clearly important that the introduction of gravity does not affect the existing scheme, other than by introducing the required body force, since terms of order $O(g)$ cannot be neglected in the fluid equations.

\section{LATTICE BOLTZMANN MODEL}

The simulation described here is performed on a $D$-dimensional regular grid with $b$ links at each grid point.
Each link has length $c$ and direction $\boldsymbol{e}_{i}, i=1, \ldots, b$. In practice the grid is either a two-dimensional hexagonal grid [5] $(D=2, b=6)$ or a four-dimensional face-centered hypercubic lattice $[17,18](D=4, b=24)$. The technique involves simulating the Boltzmann equation $[19,20]$

$$
f_{i}\left(\boldsymbol{r}+\boldsymbol{e}_{i}, t+1\right)-f_{i}(\boldsymbol{r}, t)=\Omega_{i}(\boldsymbol{r}, t) .
$$

The functions $f_{i}(\boldsymbol{r}, t), i=1, \ldots, b$ are the distribution functions along the $b$ links at position $\boldsymbol{r}$ and time $t$. The fluid density, $\rho$, and velocity, $\boldsymbol{u}$, can be found from the distribution functions as

$$
\rho=\sum_{i} f_{i} \text { and } \rho u_{\alpha}=\sum_{i} f_{i} e_{i \alpha},
$$

where we have used the notation $\left(\boldsymbol{e}_{i}\right)_{\alpha}=e_{i \alpha}$. The collision term, $\Omega_{i}(\boldsymbol{r}, t)$, is usually taken to be the single relaxation time or Bhatnagar-Gross-Krook (BGK) operator $[21,6]$

$$
\Omega_{i}(\boldsymbol{r}, t)=-\frac{1}{\tau}\left[f_{i}(\boldsymbol{r}, t)-\bar{f}_{i}(\boldsymbol{r}, t)\right],
$$

where $\bar{f}_{i}$ is the equilibrium distribution function and $\tau$ is the relaxation time, where $\tau>1 / 2$. The form of this equilibrium distribution function must be chosen so that the fluid mass and momentum are conserved and so that the resulting continuum equations describe the hydrodynamics of the fluid being simulated [12]. The correct form of the equilibrium distribution also ensures that the fluid is isotropic and Galilean invariant [22]. The following equilibrium distribution function produces an isotropic, single phase fluid that satisfies the continuity and Navier-Stokes equations:

$$
\bar{f}_{i}(\boldsymbol{r}, t)=E_{i}(\rho, \boldsymbol{u}),
$$

where 


$$
E_{i}(\rho, \boldsymbol{u})= \begin{cases}\rho\left(\frac{1-d_{0}}{b}+\frac{D}{c^{2} b} \boldsymbol{e}_{i} \cdot \boldsymbol{u}+\frac{D(D+2)}{2 c^{4} b}\left(\boldsymbol{e}_{i} \cdot \boldsymbol{u}\right)^{2}-\frac{D u^{2}}{2 c^{2} b}\right), & i=1, \ldots, b \\ \rho\left(d_{0}-\frac{u^{2}}{c^{2}}\right), & i=0\end{cases}
$$

and $d_{0}$ is a constant $[3,7]$.

\section{CHAPMAN-ENSKOG EXPANSION}

The derivation of the continuity equation and the NavierStokes equation from the equilibrium distribution is normally carried out using a Chapman-Enskog expansion, following the lattice gas derivation of Frisch et al. [17]. To perform the Chapman-Enskog expansion we must first Taylor expand Eq. (1):

$$
\begin{aligned}
f_{i}\left(\boldsymbol{r}+\boldsymbol{e}_{i}, t+1\right)-f_{i}(\boldsymbol{r}, t) \simeq & {\left[\partial_{t}+e_{i \alpha} \partial_{\alpha}+\frac{1}{2} e_{i \alpha} \partial_{\alpha}\left(e_{i \beta} \partial_{\beta}+\partial_{t}\right)\right.} \\
& \left.+\frac{1}{2} \partial_{t}\left(e_{i \alpha} \partial_{\alpha}+\partial_{t}\right)\right] f_{i}(\boldsymbol{r}, t) .
\end{aligned}
$$

Expanding the population functions and the time and space derivatives in terms of the Knudsen number [19,17], $\epsilon$, we get

$$
\begin{gathered}
f_{i}=f_{i}^{(0)}+\epsilon f_{i}^{(1)}+\epsilon^{2} f_{i}^{(2)}+\cdots, \\
\partial_{t}=\epsilon \partial_{1 t}+\epsilon^{2} \partial_{2 t}+\cdots, \\
\partial_{\boldsymbol{r}}=\epsilon \partial_{1 \boldsymbol{r}} .
\end{gathered}
$$

Substitution of Eq. (7) into Eq. (6) and considering separately the terms $O(\epsilon)$ and $O\left(\epsilon^{2}\right)$ we can perform a Chapman-Enskog expansion to obtain the continuity equation,

$$
\partial_{t} \rho+\partial_{\alpha} \rho u_{\alpha}=0
$$

and the Navier-Stokes equation

$$
\begin{aligned}
\partial_{t} \rho u_{\alpha}+\partial_{\beta} \rho u_{\beta} u_{\alpha}= & -\partial_{\beta}\left[\frac{\rho\left(1-d_{0}\right)}{D} c^{2} \delta_{\alpha \beta}\right]+\nu \partial_{\beta} \partial_{\beta} \rho u_{\alpha} \\
& +\partial_{\alpha} \zeta \partial_{\beta} \rho u_{\beta},
\end{aligned}
$$

where $\nu=c^{2}(\tau-1 / 2) /(D+2)$ and $\zeta=(\tau-1 / 2)\left[2 c^{2} /(D\right.$ $\left.+2)-c^{2}\left(1-d_{0}\right) / D\right]$ are the kinematic and bulk viscosities. The pressure term in Eq. (9) is $p=\rho c^{2}\left(1-d_{0}\right) / D$, which gives the speed of sound as

$$
c_{s}=\sqrt{\frac{c^{2}\left(1-d_{0}\right)}{D}} .
$$

\section{GRAVITY IN A LATTICE BOLTZMANN MODEL}

We now wish to consider a lattice Boltzmann model that will mimic the Navier-Stokes equation with a body force.

\section{A. The classic Boltzmann equation}

The Boltzmann equation for a fluid with a body force per unit mass $\boldsymbol{F}$ is [19]

$$
\partial_{t} f+c_{\alpha} \partial_{r \alpha} f+F_{\alpha} \partial_{c \alpha} f=\Omega(f),
$$

where $f(\boldsymbol{c}, \boldsymbol{r}, t) d \boldsymbol{c} d \boldsymbol{r}$ is the number of molecules at time $t$ with velocities in the range $\boldsymbol{c} \rightarrow \boldsymbol{c}+d \boldsymbol{c}$ and position in the range $\boldsymbol{r}$ $\rightarrow \boldsymbol{r}+d \boldsymbol{r}$ and

$$
\partial_{c \alpha}=\frac{\partial}{\partial c^{\alpha}} .
$$

The difference between the Boltzmann equation when there is no body force present and when there $i s$ a body force is an extra term: $F_{\alpha} \partial_{c \alpha} f$. In the lattice Boltzmann equation we are looking to add a similar term to incorporate a body force. Since, however, the velocity of all the "particles" is constant in the lattice Boltzmann model, we cannot simply introduce an expression with exactly the same form but must instead look to add a term that will modify the fluid momentum.

\section{B. Combining the gravity term and the pressure tensor- method (1)}

When a body force is included in the Navier-Stokes equation it is common to express the force in terms of the gravitational potential: $-\rho \boldsymbol{\nabla} \phi$. When this approach is taken, and the density variation produced by the body force is negligible, the Navier-Stokes equation incorporating the body force can be expressed in the same form as in the absence of gravity but with an altered pressure: $p \rightarrow p+\rho \phi$. Following this approach we can redefine the equilibrium distribution:

$$
E_{i}^{\prime}(\rho, \boldsymbol{u})= \begin{cases}\rho\left(\frac{1-d_{0}}{b}+\frac{l \phi D}{b c^{2}}+\frac{D}{c^{2} b} \boldsymbol{e}_{i} \cdot \boldsymbol{u}+\frac{D(D+2)}{2 c^{4} b}\left(\boldsymbol{e}_{i} \cdot \boldsymbol{u}\right)^{2}-\frac{D u^{2}}{2 c^{2} b}\right), & i=1, b \\ \rho\left(d_{0}-\frac{l \phi D}{c^{2}}-\frac{u^{2}}{c^{2}}\right), & i=0,\end{cases}
$$


with $l=1$. The parameter $l$ is introduced here in such a way that $l=1$ corresponds to gravity being incorporated as a pressure term and $l=0$ corresponds to the standard lattice Boltzmann model without gravity.

\section{Calculating the equilibrium distribution with an altered velocity-method (2)}

Gravity can be introduced into the lattice Boltzmann scheme by considering the momentum change produced by a body force [23]. If a gravitational force $\boldsymbol{F}$ is acting, then at every timestep there is a change of momentum $\Delta \boldsymbol{P}=\boldsymbol{F}$. To incorporate this into the model an equilibrium distribution

$$
\bar{f}_{i}(\boldsymbol{r}, t)=E_{i}\left(\rho, \boldsymbol{u}^{*}\right)
$$

is used where $\boldsymbol{u}^{*}$ is the "equilibrium velocity" [24], which is given by [23]

$$
\rho \boldsymbol{u}^{*}=\rho \boldsymbol{u}+\tau \boldsymbol{F} .
$$

Here $\boldsymbol{u}$ is defined, as before, by $\rho u_{\alpha}=\sum_{i} f_{i}(\boldsymbol{r}, t) e_{i \alpha}$. The fluid momentum $\rho \boldsymbol{v}$ is defined [24] to be the average of the momentum before the collision, $\rho \boldsymbol{u}$, and the momentum after the collision, $\rho \boldsymbol{u}+\boldsymbol{F}$ :

$$
\rho v_{\alpha}=\rho u_{\alpha}+\frac{1}{2} F_{\alpha} .
$$

\section{Adding an additional term to the Boltzmann equation-method (3)}

Gravity can also be introduced into the lattice Boltzmann scheme in a manner similar to that adopted for the lattice-gas model [17], that is, by adding a term to the collision function that modifies the distribution function $[25,26]$. Here the Boltzmann equation is

$$
f_{i}\left(\boldsymbol{r}+\boldsymbol{e}_{i}, t+1\right)-f_{i}(\boldsymbol{r}, t)=\Omega_{i}(\boldsymbol{r}, t),
$$

where

$$
\Omega_{i}(\boldsymbol{r}, t)=-\frac{1}{\tau}\left[f_{i}(\boldsymbol{r}, t)-\bar{f}_{i}(\boldsymbol{r}, t)\right]+\frac{D}{b c^{2}} F_{\alpha} e_{i \alpha},
$$

and $\boldsymbol{u}$ and $\bar{f}_{i}$ are defined in the usual way: $\rho u_{\alpha}=\Sigma_{i} f_{i} e_{i \alpha}$ and $\bar{f}_{i}=E(\rho, \boldsymbol{u})$. The fluid momentum is defined, as before, through $\rho v_{\alpha}=\rho u_{\alpha}+\frac{1}{2} F_{\alpha}$.

\section{E. Composite model-method (4)}

Here we consider a new method for introducing gravity into the lattice Boltzmann model. This has the collision function given by

$$
\Omega_{i}(\boldsymbol{r}, t)=-\frac{1}{\tau}\left[f_{i}(\boldsymbol{r}, t)-\bar{f}_{i}(\boldsymbol{r}, t)\right]+\frac{2 \tau-1}{2 \tau} \frac{D}{b c^{2}} F_{\alpha} e_{i \alpha},
$$

where

$$
\bar{f}=E(\rho, \boldsymbol{u}+\boldsymbol{F} / 2 \rho) .
$$

This is a combination of methods (2) and (3) with the coefficients selected to ensure the model satisfies the continuity and Navier-Stokes equations for a fluid under the influence of a body force. This will be shown in Sec. IV F.

\section{F. The equations of motion for a lattice Boltzmann model incorporating gravity}

Now consider the following Boltzmann equation:

$$
f_{i}\left(\boldsymbol{r}+\boldsymbol{e}_{i}, t+1\right)-f_{i}(\boldsymbol{r}, t)=-\frac{1}{\tau}\left(f_{i}-\bar{f}_{i}\right)+m \frac{D}{b c^{2}} F_{\alpha} e_{i \alpha},
$$

where

$$
\bar{f}_{i}=E_{i}^{\prime}(\rho, \boldsymbol{u}+n \boldsymbol{w}),
$$

$\boldsymbol{w}=\tau \boldsymbol{F} / \rho$ and $E_{i}^{\prime}$ is defined by Eq. (13). This represents method (1) for $l=1, m=n=0$, method (2) for $m=1, l=n$ $=0$, method (3) for $n=1, l=m=0$, and method (4) for $l$ $=0, m=(2 \tau-1) /(2 \tau), n=1 /(2 \tau)$. As before, we wish to perform a Chapman-Enskog expansion by expressing

$f_{i}=f_{i}^{(0)}+\epsilon f_{i}^{(1)}+\epsilon^{2} f_{i}^{(2)}, \quad \partial_{t}=\epsilon \partial_{1 t}+\epsilon^{2} \partial_{2 t}$, and $\partial_{\alpha}=\epsilon \partial_{1 \alpha}$,

where the notation $\left(\partial_{1 r}\right)_{\alpha}=\partial_{1 \alpha}$ has been used. The body force $F_{\alpha}$, and hence $\phi$, are of order $\epsilon$ [25]. This can be seen by assuming that $F_{\alpha}=O\left(\epsilon^{0}\right)$ and considering the zerothorder expansion of the Chapman-Enskog expansion:

$$
f_{i}^{(0)}=\bar{f}_{i}+\frac{m \tau D}{b c^{2}} F_{\alpha} e_{i \alpha}
$$

Multiplying this expansion by $e_{i \beta}$ and summing gives

$$
\sum_{i} f_{i}^{(0)} e_{i \beta}=\sum_{i} \bar{f}_{i} e_{i \beta}+m \tau F_{\beta}=\rho u_{\beta}+(n+m) \tau F_{\beta} .
$$

But $\Sigma_{i} f_{i} e_{i \beta}=\rho u_{\beta}$ so we must have

$$
\epsilon\left(\sum_{i} f_{i}^{(1)} e_{i \beta}+\epsilon \sum_{i} f_{i}^{(2)} e_{i \beta}+\cdots\right)=-(n+m) F_{\beta},
$$

which requires $F_{\alpha}=O(\epsilon)$ or $f_{i}^{(1)}=O\left(\epsilon^{-1}\right)$, both of which are in contradiction of the hypothesis. Thus, since $\bar{f}_{i}$ is now a function of $\boldsymbol{F}$, we also need to expand

$$
\bar{f}_{i}=\bar{f}_{i}^{(0)}+\epsilon \bar{f}_{i}^{(1)}+\epsilon^{2} \bar{f}_{i}^{(2)}, \quad F_{\alpha}=\epsilon F_{1 \alpha}, \text { and } \phi=\epsilon \phi_{1} .
$$

Performing a Chapman-Enskog expansion, see Appendix A, we obtain the following macroscopic equations:

$$
\partial_{t} \rho+\partial_{\alpha}\left[\rho u_{\alpha}+\frac{1}{2}(n+m) F_{\alpha}\right]=0
$$

and 


$$
\begin{aligned}
\partial_{t}\left[\rho u_{\alpha}+\frac{1}{2}(n+m) F_{1 \alpha}\right]+\partial_{\beta}\left[\rho u_{\alpha} u_{\beta}+\tau n\left(u_{\alpha} F_{\beta}+u_{\beta} F_{\alpha}\right)\right] \\
=-\partial_{\alpha}\left(\frac{\rho\left(1-d_{0}\right) c^{2}}{D}\right)+\nu \partial_{\beta} \partial_{\beta} \rho u_{\alpha}+\zeta \partial_{\alpha} \partial_{\beta} \rho u_{\beta} \\
\quad+(n+m) F_{\alpha}-l \partial_{\alpha} \rho \phi
\end{aligned}
$$

For method (1) we have $l=1, m=n=0$ in which case Eqs. (28) and (29) are the continuity and Navier-Stokes equations for a fluid with velocity $\boldsymbol{u}$ and a body force $\boldsymbol{F}=-\rho \boldsymbol{\nabla} \phi$. For methods (2), (3), and (4) we have $l=0, m+n=1$ in which case Eqs. (28) and (29) can be written (up to second order in $\epsilon)$

$$
\partial_{t} \rho+\partial_{\alpha} \rho v_{\alpha}=0
$$

and

$$
\begin{aligned}
& \partial_{t} \rho v_{\alpha}+\partial_{\beta} \rho v_{\alpha} v_{\beta}+\partial_{\beta}(n \tau-1 / 2)\left(u_{\alpha} F_{\beta}+u_{\beta} F_{\alpha}\right) \\
& \quad=-\partial_{\alpha}\left(\frac{\rho\left(1-d_{0}\right) c^{2}}{D}\right)+\nu \partial_{\beta} \partial_{\beta} \rho v_{\alpha}+\zeta \partial_{1 \alpha} \partial_{1 \beta} \rho v_{\beta}+F_{\alpha},
\end{aligned}
$$

where $\boldsymbol{v}$ is defined, as before, to be the mean fluid velocity: $\rho v_{\alpha}=\rho u_{\alpha}+F_{\alpha} / 2$. Equations (30) and (31) are the continuity and the Navier-Stokes equations for a fluid with velocity $\boldsymbol{v}$ and a body force $\boldsymbol{F}$ with an additional term $\partial_{\beta}(n \tau$ $-1 / 2)\left(u_{\alpha} F_{\beta}+u_{\beta} F_{\alpha}\right)$. This term may be small for the values of $\boldsymbol{F}$ considered here but will only be zero for $n=1 /(2 \tau)$, that is for method (4).

\section{G. Review of methods}

The Navier-Stokes equation is recovered from Eq. (29) for $l=1$ and $m=n=0$, only when the term $\partial_{\alpha} \rho \phi$ can be expressed as $\rho \partial_{\alpha} \phi$. Thus we only expect method (1) to be appropriate in situations where there is no density change across the fluid, or the density change is negligibly small. This is common to any situation where gravity is introduced through a potential that modifies the pressure term. Methods (2) and (3) can be applied where there is a density change but we are looking at a steady state solution, where $\partial_{\beta}\left(u_{\alpha} F_{\beta}\right.$ $\left.+u_{\beta} F_{\alpha}\right)=0$. Two situations were this can occur are considered in this paper. First, when the steady state velocity is zero and second, when the nonlinear term in the NavierStokes equation is zero and the induced velocity is parallel to $\boldsymbol{F}$. Method (4) is applicable in any situation where gravity is applied in the incompressible limit. In general the variation between methods (2), (3), and (4) will depend on the values of $\boldsymbol{u}$ and $\boldsymbol{F}$ through the anomalous term $\partial_{\beta}\left(u_{\alpha} F_{\beta}+u_{\beta} F_{\alpha}\right)$. The difference between the lattice Boltzmann operators $\Omega_{i}^{(2)}-\Omega_{i}^{(3)}$ for methods (2) and (3), acting on the same distribution function $f_{i}$, is

$$
\begin{aligned}
& \frac{\rho}{\tau}\left\{\left[\frac{2 u_{\alpha} \tau F_{\beta}}{\rho}+\frac{\tau^{2} F_{\alpha} F_{\beta}}{\rho^{2}}\right] \frac{D(D+2) e_{i \alpha} e_{i \beta}}{2 c^{4} b}\right. \\
& \left.-\frac{D}{2 c^{2} b}\left[\frac{2 u_{\alpha} \tau F_{\alpha}}{\rho}+\frac{\tau^{2} F_{\alpha} F_{\alpha}}{\rho^{2}}\right]\right\} i=1, b \\
& -\frac{\rho}{\tau}\left(\frac{2 \tau F_{\alpha} u_{\alpha}}{\rho c^{2}}+\frac{\tau^{2} F_{\alpha} F_{\alpha}}{\rho^{2} c^{2}}\right), \quad i=0 .
\end{aligned}
$$

The difference contains terms $O(u F / \rho)$ and $O\left((F / \rho)^{2}\right)$. It is assumed in the derivation of the equations of motion that $\boldsymbol{u}$ $\ll c_{s}$ and, in the incompressible limit, we also have $|F|$ $\ll c_{s}^{2} / z$. This gives a measure on the size of the terms in Eq. (32). Thus, in general, the difference expressed in Eq. (32) will be small. Although $F / \rho$ is small it can still produce a significant effect. The difference expressed in Eq. (32) being small does not imply that the density change is small, as required for method (1). The differences $\Omega_{i}^{(2)}-\Omega_{i}^{(4)}$ and $\Omega_{i}^{(3)}-\Omega_{i}^{(4)}$ will contain terms of the same order. We note that while the values of $\Omega_{i}^{(1)}-\Omega_{i}^{(2)}$ are different for each $i$, $\Sigma_{i}\left(\Omega_{i}^{(1)}-\Omega_{i}^{(2)}\right)=0$ and $\Sigma_{i}\left(\Omega_{i}^{(1)}-\Omega_{i}^{(2)}\right) e_{i \gamma}=0$. This means that in simulations where methods (2), (3), and (4) satisfy the same equations of motion, that is $\partial_{\beta}\left(u_{\alpha} F_{\beta}+u_{\beta} F_{\alpha}\right)=0$, there will be a difference in the values of $f_{i}$ between the different models, but the values of $\rho$ and $\boldsymbol{u}$ should, however, be identical.

\section{ERRORS IN A LATTICE BOLTZMANN SIMULATION}

There are a number of sources of error that can affect a lattice Boltzmann simulation. Rounding errors will always be present in any numerical model. Here double precision arithmetic was used to give results with 15 significant figures. In single precision simulations a precision of seven significant figures would give an error of $O\left(10^{-7}\right)$.

In the derivation of the Navier-Stokes equation described in Sec. III only terms up to $O\left(\epsilon^{2}\right)$ are considered-higher order terms are neglected. The Knudsen number, $\epsilon$, is the ratio of the grid separation to the typical macroscopic length in the simulation. Thus we must ensure that $\epsilon$ is small in any simulation to minimize the error introduced by neglecting higher order terms. Small $\epsilon$ is equivalent to having a large number of grid points corresponding to the shortest length scale in the simulation. Here we typically use a grid with 64 points, which gives $\epsilon=1 /(64 \sqrt{3} / 2)$, since the length scale is the grid size and the grid is orientated so that the horizontal separation of grid points is $\sqrt{3} / 2$. This also relates to the spatial discretization error, which is introduced because we are mimicking a continuous system by a grid simulation. A small Knudsen number that implies a significant number of grid points along any length scale also implies a small spatial discretization error. Time is also discrete in the lattice Boltzmann model and the temporal discretization error must also be small if a simulation is to produce meaningful results. That is, a typical macroscopic time scale must be large with respect to the discrete time step. Since $c_{s}$ is $O(1)$ this is also satisfied by a small Knudsen number.

It has been shown by many authors that, provided the boundary conditions are suitably implemented, the lattice 
Boltzmann model is a second-order scheme. This was studied by Noble et al. [27] who considered flow between two parallel porous plates; one stationary and one moving. They showed that the lattice Boltzmann model applied with the boundary conditions used here is a second-order scheme. For a length scale of $64 \sqrt{3} / 2$ lattice units they find the average error to be $E \simeq 3 \times 10^{-4}$, where $E$ is defined by

$$
E=\frac{\sum_{y}|u-\hat{u}|}{\sum_{y}|u|},
$$

and where $u$ is the simulated velocity, $\hat{u}$ is the analytic velocity, and the summation is over all points in a line perpendicular to the two plates. This gives an estimation of the discretization errors for a simulation with length scale $64 \sqrt{3} / 2$.

We note that the lattice Boltzmann equation, Eq. (1), can be viewed as a finite difference equation. Although the discretization is first order the lattice Boltzmann method is a second-order scheme as discussed above. The second-order nature of the lattice Boltzmann model is further discussed by Sterling and Chen [28]. Under certain special circumstances a second-order difference scheme on a regular grid can give an exact solution with zero discretization error. This occurs if the terms in the expression for the discretization error are identically zero. One such case is Poiseuille flow, which is created between stationary parallel vertical walls when a fluid is driven by gravity. The steady-state solution can easily be found since the Navier-Stokes equation reduces to

$$
\nu \frac{\partial^{2} u_{z}(x)}{\partial x^{2}}=g,
$$

which has the solution

$$
u_{z}(x)=\frac{g}{2 \nu}\left(x^{2}-L^{2}\right)
$$

where the walls are at $x= \pm L$. The truncation error for a second-order central difference scheme depends on the derivatives $\partial^{4} u_{z}(x) / \partial x^{4}, \partial^{6} u_{z}(x) / \partial x^{6}, \ldots$, which are all zero in this special case. Thus we expect to be able to simulate Poiseuille flow using the lattice Boltzmann model to within the truncation error of the computer [27].

The lattice Boltzmann model satisfies the Navier-Stokes equation in the nearly incompressible limit. By introducing gravity we inevitably introduce a compressibility error into the system. Here we consider a body force in the incompressible limit, $g z \ll c_{s}$, and consider the compressibility errors that this introduces into the model.

\section{NUMERICAL SIMULATIONS}

Methods (1)-(4) were implemented so that the affect they have on a fluid simulation could be observed and any differences between the models could be considered. The value of $d_{0}=0.5$ was used throughout. The simulations were written in FORTRAN using double precision arithmetic giving 64-bit

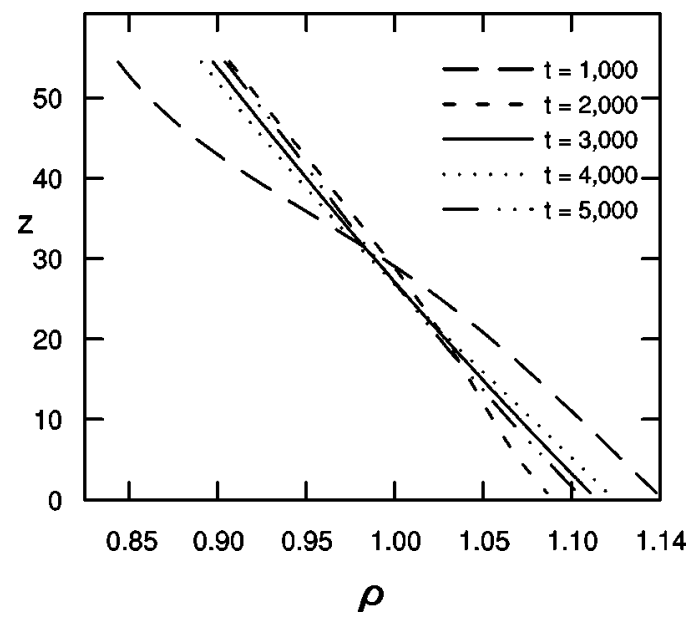

FIG. 1. Density as a function of height at selected times when gravity is applied using method (3) when $\boldsymbol{F}=-0.001 \boldsymbol{e}_{z}$. The density is measured in particles per site and the height in lattice sites.

precision. The single phase simulations were performed on a Sun server, each simulation taking no more than $20 \mathrm{CPU}$ minutes. The immiscible fluid simulations were performed on the CM-200 at Edinburgh University.

\section{A. Density gradient}

A system was initialized on a $64 \times 64$ grid with zero velocity and initial density $\rho_{0}=1$. An impermeable boundary was placed at the bottom $(z=0)$ of the grid, which also acted as a boundary at the top; continuous boundary conditions were applied at the other two edges. Gravity was then applied using each of the methods and the density measured every 1000 timesteps along a vertical line through the middle of the grid. The results are shown in Fig. 1 at times 1000, 2000, 3000, 4000, and 5000 timesteps when gravity is applied with strength $g=0.001$ using method (3) with $\tau=1.0$. The density profile is seen to "oscillate" about its final position for several thousand timesteps before reaching its final state. The final state density distribution is found to lie close to the distribution for $t=3000$ timesteps in Fig. 1 and so is not included for clarity. The observed "oscillations"' are just the damping of sound waves due to the fact that the initial density is uniform.

This was repeated for each of the methods for $g=0.001$, $g=0.0001$, and $g=0.00001$ and for $\tau=0.55,1.0,5.5$, and 50.5. The simulations were run until the final density variation with depth settled down to a steady state. The different density variations were then compared with each other and with the analytic expressions for the incompressible limit, which are found in Appendix B. The density difference $\rho_{a b}$ for $a, b \in\{1,2,3,4, T\}$ is defined to be $\rho_{a b}=\rho^{(a)}-\rho^{(b)}$, where $\rho^{(a)}$ is the steady state density produced when method (a) is applied for $a=1,2,3$, and 4 and the density predicted by the theoretical expression, Eq. (B19), for $a=T$. First we consider the differences between method (1) and method (4). This is shown in Fig. 2 when $g=0.001$ and there is a significant density change across the fluid of about $20 \%$. The density variation produced by method (4) is seen to be approximately linear while for method (1) it is curved. This is expected since the conditions here clearly break the assump- 


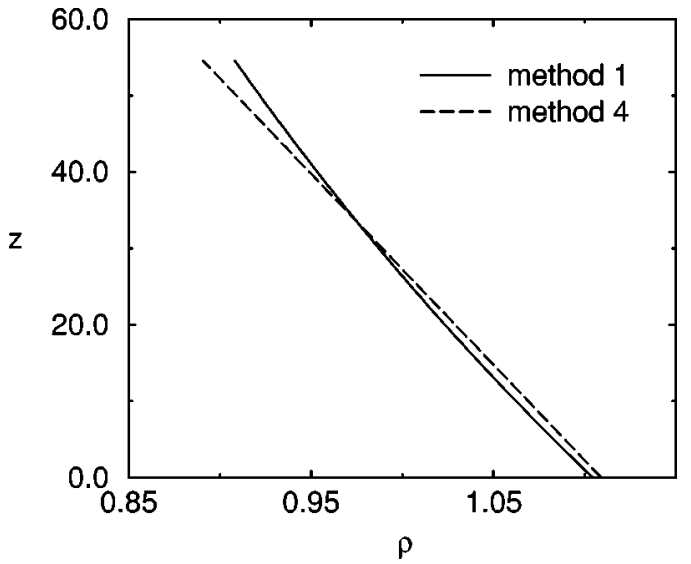

FIG. 2. The density variations with height produced by methods (1) and (4) when $g=0.001$. The density is measured in particles per site and the height in lattice sites.

tion that $\rho$ is constant as required in method (1). When $g$ $=0.00001$ and the density variation is about $0.2 \%$, the density variation is considerably smaller and so is the difference between the density variation produced by methods (1) and (4) as shown in Fig. 3. The difference here is not significantly larger than the computational rounding error in a standard 32-bit calculation. When $g=0.0001$ the density variation across the fluid is about $2 \%$ and the difference between the results, $\left|\rho_{14}\right|=O\left(10^{-3}\right)$. The difference between the density variation produced by method (4) and the analytic results in the incompressible limit, $g z / c_{s}^{2} \ll 1$, is shown in Fig. 4 for the three values of $g$ considered. The ratio $c_{s}^{2} / g z$ is $4.5,45$, and 450 for $g=0.001,0.0001$, and 0.00001 , respectively. For the lowest value of $g$ the incompressibility condition is fully satisfied and the variations are not much larger than the numerical rounding error when standard 32-bit precision is used. When $g=0.001$ the incompressibility condition $\left(g z / c_{s}^{2} \ll 1\right)$ is just met since $g z / c_{s}^{2}=0.022$. Here the agreement between the analytical results and the simulation is reasonable with a variation of no more than about $0.01 \%$. This is no larger than the typical discretization error of $O\left(10^{-4}\right)$, which we would expect in a simulation of this size. For the largest value of $g$ the incompressible limit is not truly satisfied $\left(g z / c_{s}^{2}=0.22\right)$, however, even here the varia-

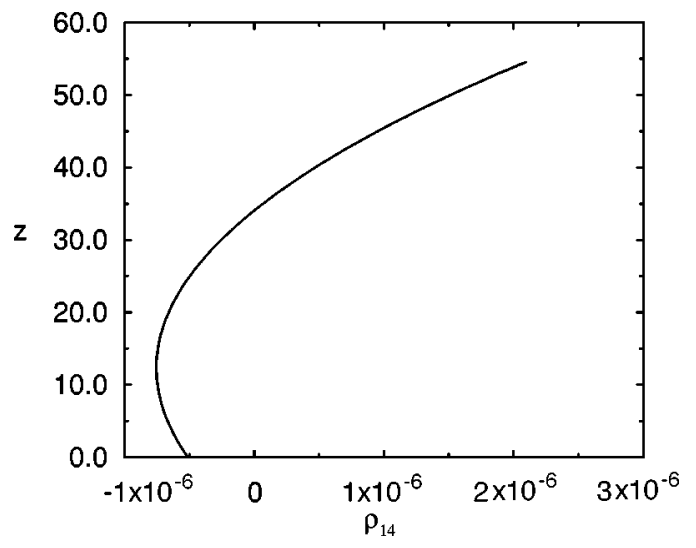

FIG. 3. The difference between the density variations with height produced by methods (1) and (4) when $g=0.00001$. The height is measured in lattice sites.

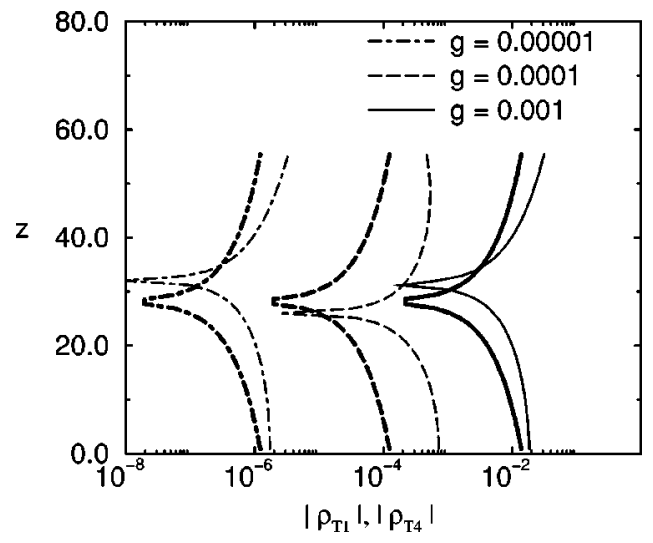

FIG. 4. The density differences $\left|\rho_{T 1}\right|$ and $\left|\rho_{T 4}\right|$ as functions of depth for different values of $g$. The differences $\left|\rho_{T 1}\right|$ are represented by the thin lines and the differences $\left|\rho_{T 4}\right|$ by the thick lines. The height is measured in lattice sites.

tion is only about $1 \%$. The difference between the results for method (1) and the analytic result are also shown in Fig. 4. In each case the difference is generally larger. For $g$ $=0.00001$ it is $O\left(10^{-6}\right)$, as was seen in Fig. 3 and is not significant. For $g=0.0001$ the error is $O\left(10^{-3}\right)$, which is an order of magnitude larger then $\left|\rho_{T 4}\right|$ suggesting that the constant density approximation is not valid and method (4) [or methods (2) or (3)] would be preferred. For $g=0.001$ the difference $\left|\rho_{T 1}\right|$ can be larger than $1 \%$ and is typically double $\left|\rho_{T 4}\right|$ although the differences are for different reasons. The large value of $\left|\rho_{T 1}\right|$ is due to the density gradient that cannot be approximated to zero. The error in $\left|\rho_{T 4}\right|$ is due to $g z=O\left(c_{s}^{2}\right)$ implying that we are outside the incompressible limit and so we do not expect a linear density change. For both methods the difference between the simulation results and theory is seen to depend on $g$, suggesting that in this case the compressibility error is the main source of error.

The difference between the densities predicted by methods (2), (3), and (4) are shown in Fig. 5. For each value of $g$ the densities obtained by methods (2) and (4) and hence the difference $\left|\rho_{24}\right|$ are independent of $\tau$ as predicted by Eq.

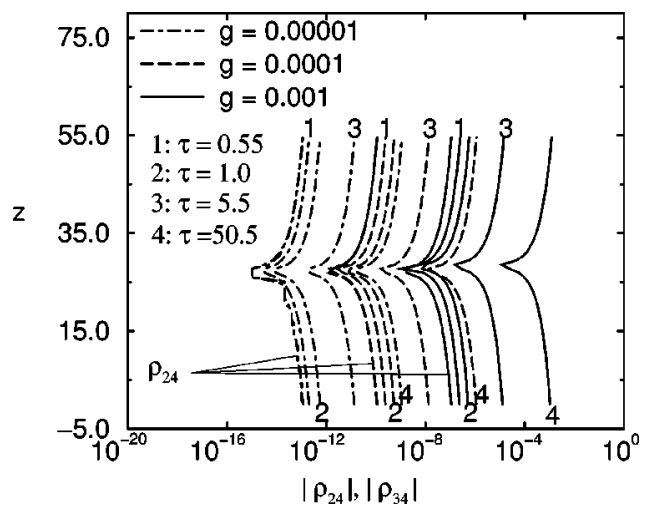

FIG. 5. The density differences $\left|\rho_{24}\right|$ and $\left|\rho_{34}\right|$ as functions of depth for different values of $g$ and $\tau$. The differences $\left|\rho_{24}\right|$ are independent of $\tau$ so, for a given value of $g$ the four results for the four different values of $\tau$ lie on the same curve. The other curves represent $\left|\rho_{34}\right|$ and are marked $1,2,3$, or 4 corresponding to $\tau$ $=0.55, \tau=1.0, \tau=5.5$, and $\tau=50.5$, respectively. The height is measured in lattice sites. 
(B18). The differences $\left|\rho_{34}\right|$ are increasing functions of $\tau$, in agreement with the difference being dependent on a term containing the factor $(\tau-1 / 2)$, for $\tau>1 / 2$. All the relative differences are observed to increase with $g$. The difference between the results for $g=0.00001$ and $g=0.0001$ and the difference between the results for $g=0.0001$ and $g=0.001$ differing by a factor $O\left(10^{-3}\right)$. In all the cases considered the largest value of the differences between the methods is at least an order of magnitude smaller than the difference between the results and the analytic expression, Eq. (B19). For the lower values of $g(0.0001$ and 0.00001$)$, which are strictly within the incompressible regime, the difference is greater and at the low values of $\tau$ at which the lattice Boltzmann model is normally run, the difference is greater still: for $g=0.0001$ and $\tau=1$ we have $\left|\rho_{34}\right|=O\left(10^{-9}\right)$ and $\left|\rho_{T 4}\right|$ $=O\left(10^{-4}\right)$. Thus the density gradients produced by the different models are not significantly different when compared to the error introduced by the incompressibility approximation.

\section{B. Simulations with nonzero velocities}

Two situations are considered where the fluid velocity is nonzero. Gravity driven Poiseuille flow, where the density is constant, the velocity is nonzero, and the nonlinear term in the Navier-Stokes equation is zero, is a simulation that all four methods should be suited to. During the time between the initialization of the fluid as described in Sec. VI A and the formation of the steady state density gradients that were measured, the density and velocity of the fluid are functions of time, as seen in Fig. 1, in such a way that the nonlinear term in the Navier-Stokes equation is nonzero. Thus the differences between methods (2), (3), and (4) should be observable in this interim period.

\section{Poiseuille flow}

Poiseuille flow is created between stationary parallel vertical walls when a fluid is driven by gravity and has a steady state solution

$$
u_{z}(x)=\frac{g}{2 \nu}\left(x^{2}-L^{2}\right)
$$

where the walls are at $x= \pm L$. Method (2) has been applied to simulate Poiseuille flow [23] for a body force that produces a maximum velocity 0.0005 and method (3) has been applied [25,27] and seen to produce results that are correct up to the machine accuracy. Poiseuille flow was simulated here for all four methods. When suitable boundary conditions [27] were applied at the wall boundaries and continuous boundary conditions at the nonwall edges, methods (2), (3), and (4) all produced the expected flow pattern correct to machine accuracy for a range of different values of $\tau$ and $g$. Since method (4) can be thought of as a composite of methods (2) and (3) it is hardly surprising that it performs equally well. Method (1) can also be applied here since there is no density variation in the fluid. Since the gravitational potential is a linear function of the vertical position continuous boundary conditions cannot be applied across the open ends; this would produce a large potential difference across the edge of the grid that would exactly cancel out the gravitational po-

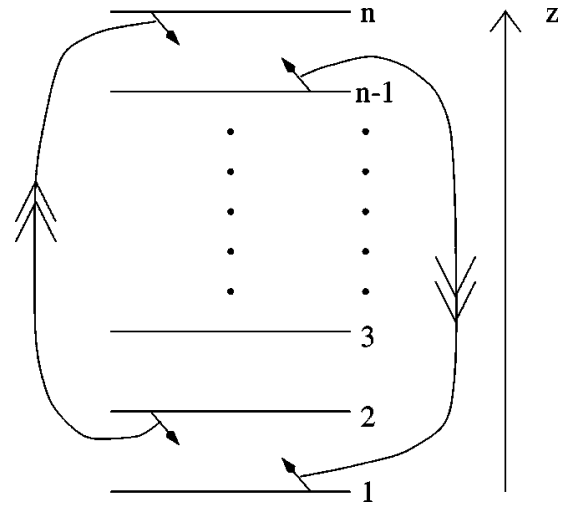

FIG. 6. The boundary condition applied to produce continuous boundary conditions for method (1).

tential. To overcome this, the following boundary conditions, see Fig. 6, were applied after the standard streaming: distribution functions on the penultimate rows are mapped onto the end rows at the opposite side of the grid. An example of the flow patterns set up is shown in Fig. 7, which agrees with Eq. (36) to the machine accuracy. Here there is no discretization error, as discussed in Sec. V and there is no compressibility error since the density is constant.

\section{Changeable flow}

Flow phenomena where the nonlinear term of the NavierStokes equation is nonzero are in general more complex and do not have simple analytic solutions. We expect methods (2), (3), and (4) to exhibit differences in such situations so we consider the evolution of the fluid between the initialization described in Sec. VI A (zero velocity and constant density) and the steady state situation, which was just discussed. The difference between the density and velocity at a point in the center of the grid, found using methods (2), (3), and (4), as a function of time, is shown in Fig. 8 when $\tau=50.5$ and $g=0.0005$. This gives $c_{s}^{2} / g z=9$ and so is the largest value of $g$ that could be reasonably used in the incompressible limit. This value of $g$ gives a maximum value for the velocity no larger than 0.1 . The density difference, $\rho_{a b}$ between methods (a) and (b) can be thought of as relative density differences since $\rho \simeq 1$ at the center of the grid. Relative velocity differences cannot sensibly be considered since the velocity is os-

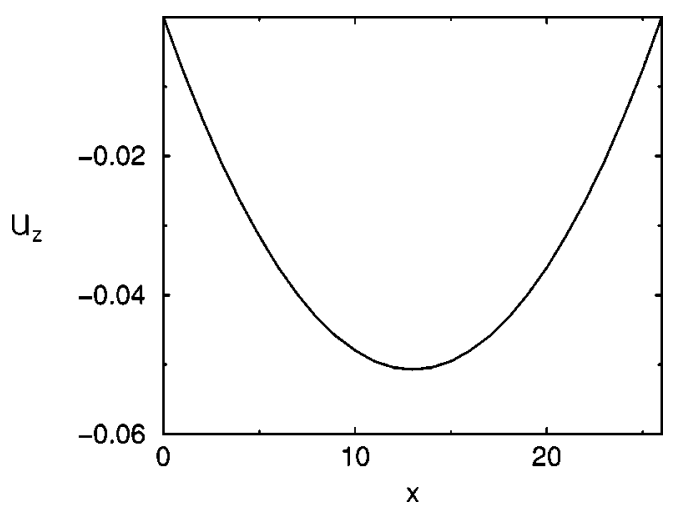

FIG. 7. The steady state Poisseuille flow simulated using method (1) when $g=0.0001$. The pipe width $x$ is measured in lattice sites and the velocity $u_{z}$ in (lattice sites)/(time step). 


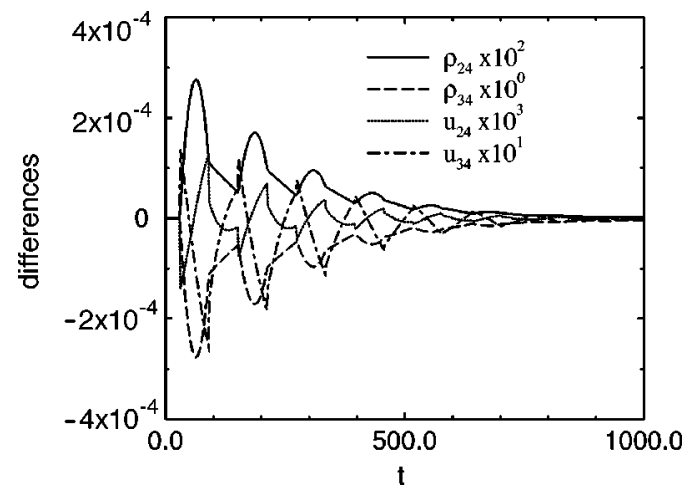

FIG. 8. The density and velocity differences $\rho_{24}, \rho_{34}, u_{24}$, and $u_{34}$ for $g=0.0005$ and $\tau=50.5$. The time is measured in time steps.

cillating about zero, however the velocity differences relative to the maximum value of the velocity can be considered. At small times $\left|\rho_{34}\right|$ peaks at about $3 \times 10^{-4}$ giving a relative density difference of about $3 \times 10^{-4}$, whereas $\left|\rho_{24}\right|$ gives a much smaller maximum density difference of about 3 $\times 10^{-6}$. Initially the values of $\left|u_{24}\right|$ and $\left|u_{34}\right|$ peak at values $O$ (10) smaller than the corresponding density differences. Since $u_{\max }=O(0.1)$ at these times the relative density difference and the relative velocity difference (relative to the maximum value of $u$ ) are of the same order for each comparison. At later times the peak values of $\left|u_{24}\right|$ and $\left|u_{34}\right|$ remain $O(10)$ smaller than the corresponding densities, however, the peak values of the velocity are reduced at these times so the relative velocity differences become larger than the relative density differences at the peaks as time increases. The absolute value of all the differences, however, decrease with time in an approximately exponential manner at about the same rate.

This was repeated for $\tau=5.50,1.0,0.55$, and 0.505 . In each case the maximum values of $\left|\rho_{a b}\right|$ and $\left|u_{a b}\right|$ were found for the different values of $a$ and $b$ of interest. The results are shown in Fig. 9 as a function of the viscosity, an increasing function of $\tau$. For both the density and the velocity, the differences between methods (2) and (4) are decreasing functions of $\tau$ and the differences between methods (3) and (4) are increasing functions of $\tau$. At $\tau=1(\nu=1 / 8)$ we find

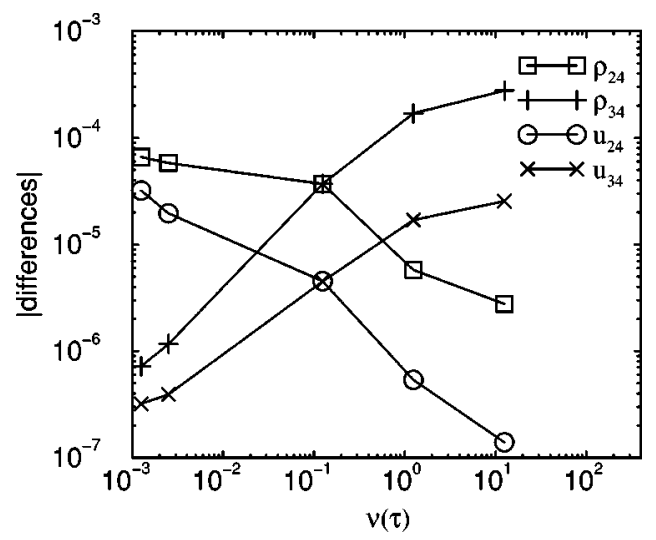

FIG. 9. The maximum values of the density and velocity differences $\rho_{24}, \rho_{34}, u_{24}$, and $u_{34}$ for $g=0.0005$ as a function of the fluid viscosity, which is an increasing function of $\tau$. The viscosity is measured in units of (lattice sites) $)^{2} /($ time step). $\left|\rho_{34}\right|=\left|\rho_{24}\right|$ and $\left|u_{34}\right|=\left|u_{24}\right|$ as predicted.

The results here show that there is an observable difference between the models, due to the incorrect nonlinear term in methods (2) and (3). The maximum value of the velocity in the simulations is at the upper end of the range of velocities that can be used in a lattice Boltzmann simulation and the value of $g$ used is at the upper limit of values for which the incompressible limit holds (at least for the value of $z$ used here). Thus we expect that the magnitude of the errors produced due to methods (2) and (3) not satisfying the correct Navier-Stokes equation will typically be no larger than the differences measured here. The results suggest that, although the differences are small, at high values of $\tau$ it is inadvisable to use method (3), while at low values of $\tau$ it is inadvisable to use method (2). At intermediated values, for example $\tau$ $=1$, the results differ by about $0.01 \%$, which is too small to make a significant difference to a simulation but the accuracy can be improved by using method (4). At low $\tau$ the difference $\rho_{24}$ is $O\left(10^{-4}\right)$ and at high $\tau$ the difference $\rho_{34}$ is $O\left(10^{-4}\right)$. These are of the same order of magnitude as the estimation of the compressibility error for $g=0.0001$, see Fig. 4, and the estimation of the discretization error, see Sec. $\mathrm{V}$. Therefore we do not expect errors due to the anomalous term in Eq. (31) to swamp the results, however, in some simulations it may be the largest source of error and so it is advisable to reduce it by using method (4).

\section{Gravity in an immiscible fluid model}

We now consider the body force applied to an immiscible binary fluid. This was done using the model of Orlandini et al. [29], where the fluid is described in terms of the total density $\rho$ and the density difference between the two fluids $\Delta \rho$. This was done using method (3) for the relaxation times $\tau_{\Delta}=0.8, \tau_{\rho}=0.9, \rho_{0}=1$ and for an interaction strength $\lambda$ $=1.1$. The immiscible binary fluid was initialized with the two fluids separated by a horizontal interface. The upper fluid has $\Delta \rho$ negative. Gravity was applied to both fluids with strength $\rho g=\left[\left(g_{a}(\rho-\Delta \rho)+g_{b}(\rho+\Delta \rho)\right] / 2\right.$, where $g_{a}$ $<g_{b}$. At temperature $T=0.5$ we expect that $|\rho / \Delta \rho| \simeq 2$ in the absence of gravity [29]. Figure 10 shows the value of the modulus of the ratio $\rho / \Delta \rho$ at different depths for the immiscible fluid when $g_{a}=0.0001$ and $g_{b}=0.0002$. At the interface the value of $|\rho / \Delta \rho|$ is different from 2.0 by no more than $4 \%$. Away from the interface the ratio appears constant with depth, and hence also with density. Thus the value of $g$ in both fluids is given by

$$
\begin{aligned}
& g_{1}=\left[g_{a}(1+1 / 2)+g_{b}(1-1 / 2)\right] / 2, \\
& g_{2}=\left[g_{a}(1-1 / 2)+g_{b}(1+1 / 2)\right] / 2 .
\end{aligned}
$$

Figure 11 shows the variation in density with depth for the immiscible fluid for two different sets of values $g_{a}$ and $g_{b}$. The values are shown in Table I as are the values of $g_{1}, g_{2}$ in the upper and lower fluids, respectively. Straight lines with gradients $4 \rho_{0} g$ are also shown in Fig. 11. The agreement between the actual gradients and the predicted gradients is good and reinforces the use of Eq. (37) for calculating $g_{1}$ and $g_{2}$. Method (3) was preferred over methods (2) and (4) here since the nonlinear term of the Navier-Stokes equa- 


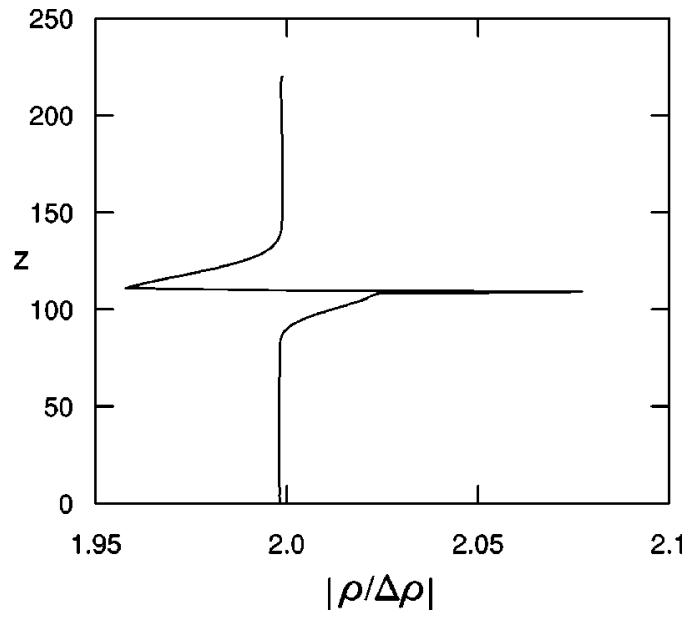

FIG. 10. The modulus of the ratio $\rho / \Delta \rho$ as a function of depth when gravity is applied to a binary fluid with a horizontal interface between the fluids. Gravity was applied with $g_{a}=0.0001$ and $g_{b}$ $=0.0002$. The height is measured in lattice sites.

tion is zero and it is not clear how replacing $\bar{f}(\rho, \boldsymbol{u})$ with $\bar{f}\left(\rho, \boldsymbol{u}^{*}\right)$ would affect the thermodynamical properties of the fluid mixture, and over method (1) since it can be applied for a larger range of $g$.

Other schemes have also been proposed for multifluid simulation. Shan et al. $[11,24]$ consider a model in which the fluids are separated by an intersite force that is introduced in the same manner as the body force in method (2). For such a model there is no thermodynamical equilibrium to be affected so method (2) can be applied [23], however, given the errors involved in method (2), particularly at low $\tau$, method (4) might be better applied in such cases.

\section{CONCLUSION}

Different methods have been considered for introducing a body force, in the incompressible limit, into the lattice Boltzmann model. Method (1) introduces gravity by including an additional term in the equilibrium distribution function,

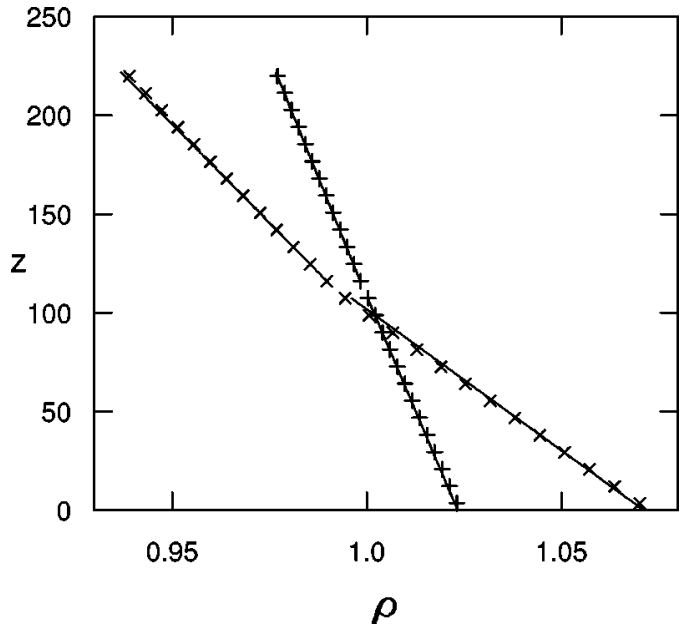

FIG. 11. The density as a function of depth for case (i) $(\times)$ and case (ii) $(+)$ shown in Table I. Also shown are straight lines with gradients $4 \rho_{0} g_{i}$. The density is measured in particles per site and the height in lattice sites.
TABLE I. The values of $g_{a}$ and $g_{b}$ used in cases (i) and (ii) in Fig. 11 and the values of $g_{1}$ and $g_{2}$ calculated using Eq. (37).

\begin{tabular}{ccccc}
\hline \hline Case & $g_{a}$ & $g_{b}$ & $g_{1}$ & $g_{2}$ \\
\hline (i) & $1 \times 10^{-4}$ & $2 \times 10^{-4}$ & $1.25 \times 10^{-4}$ & $1.75 \times 10^{-4}$ \\
(ii) & $5.0 \times 10^{-5}$ & $5.5 \times 10^{-5}$ & $5.125 \times 10^{-5}$ & $5.375 \times 10^{-5}$ \\
\hline
\end{tabular}

which produces an altered pressure term-the difference corresponding to a gravitational potential. This approach is restricted to simulations where there is no density change or the density change is small enough that it can be neglected. Method (1) was seen to be capable of simulating Poiseuille flow (where there is no density change) correct to the computer accuracy. When a density gradient is present, method (1) performs less well than the other methods considered, even when the density variation is small. The difference between the accuracy of method (1) and the other methods can be as large as an order of magnitude when $g$ is within the incompressible limit. Despite this the error was observed to be little more than the computer accuracy of a 32-bit simulation when the density variation across the fluid was $0.2 \%$.

Method (2) introduces gravity into the lattice Boltzmann model by considering the equilibrium distribution function to be a function, not of the lattice Boltzmann velocity $\left(\Sigma_{i} f_{i} \boldsymbol{e}_{i} / \Sigma_{i} f_{i}\right)$, but of an "equilibrium velocity" defined as the sum of the lattice Boltzmann velocity and $\tau \boldsymbol{F} / \rho$, where $\boldsymbol{F}$ is the body force due to gravity. Method (3) introduces gravity by adding a term to the collision function, which is proportional to $F_{\alpha} e_{i \alpha}$. For both these methods, if we define the fluid velocity, $\boldsymbol{v}$, to be the sum of the lattice Boltzmann velocity and $\boldsymbol{F} / 2 \rho$, then the models satisfy (up to second order) the continuity equation and an equation similar to the Navier-Stokes equation. The difference is an additional term of order $O(F u)$. Method (4) introduces gravity by considering the equilibrium distribution to be a function of an altered velocity and by adding an additional term to the collision operator. In this method the introduction of gravity can be thought of as being made up from a combination from method (2) and a combination from method (3). The relative strengths of the contributions are selected to ensure that the technique satisfies the exact (up to second order) NavierStokes equation in the velocity $\boldsymbol{v}$. Within the incompressible limit methods (2), (3), and (4) were found to compare well with theory in situations where the nonlinear term of the Navier-Stokes equation is zero. When the nonlinear term is nonzero an observable difference is found between methods (2) and (3), which have the wrong nonlinear term, and method (4), which has the correct nonlinear term. This difference is small but under certain circumstances is large enough to influence the simulation results, in which case method (4) is an improvement over the other methods.

The introduction of gravity into a two-phase model was also considered. Here the error introduced by the incorrect nonlinear term when method (3) is used has to be weighed against any effect of using an altered "equilibrium velocity" rather than the true velocity in the definition of $\bar{f}_{i}$ may have on the thermodynamical properties of the fluid.

\section{ACKNOWLEDGMENTS}

The authors wish to thank Dominique d'Humières for helpful comments on an earlier draft of this manuscript and 
in particular for suggesting we consider the analytical results. We are also grateful to the Edinburgh Parallel Computing Center (EPCC) for providing us with the use of their facilities.

\section{APPENDIX A: CHAPMAN-ENSKOG EXPANSION FOR A LATTICE BOLTZMANN MODEL INCORPORATING GRAVITY}

Here we wish to consider the Chapman-Enskog expansion of the Boltzmann equation

$$
f_{i}\left(\boldsymbol{r}+\boldsymbol{e}_{i}, t+1\right)-f_{i}(\boldsymbol{r}, t)=-\frac{1}{\tau}\left(f_{i}-\bar{f}_{i}\right)+m \frac{D}{b c^{2}} F_{\alpha} e_{i \alpha},
$$

where

$$
\bar{f}_{i}=E_{i}^{\prime}(\rho, \boldsymbol{u}+n \boldsymbol{w}),
$$

$\boldsymbol{w}=\tau \boldsymbol{F} / \rho$ and $E_{i}^{\prime}$ is defined by Eq. (13) where we expand

$$
f_{i}=f_{i}^{(0)}+\epsilon f_{i}^{(1)}+\epsilon^{2} f_{i}^{(2)}, \quad \partial_{t}=\epsilon \partial_{1 t}+\epsilon^{2} \partial_{2 t}, \quad \partial_{\alpha}=\epsilon \partial_{1 \alpha},
$$

$$
\bar{f}_{i}=\bar{f}_{i}^{(0)}+\epsilon \bar{f}_{i}^{(1)}+\epsilon^{2} \bar{f}_{i}^{(2)}, \quad F_{\alpha}=\epsilon F_{1 \alpha}, \quad \text { and } \phi=\epsilon \phi_{1} .
$$

Substituting the expansion of $F_{\alpha}$ and $\phi$ into $E^{\prime}$ gives

$$
E^{\prime}\left(\rho, \boldsymbol{u}+\epsilon \frac{n \tau}{\rho} \boldsymbol{F}_{1}\right)=\left\{\begin{array}{l}
\rho\left[\frac{1-d_{0}}{b}+\frac{\epsilon l \phi_{1} D}{b c^{2}}+\frac{D}{c^{2} b} e_{i \alpha} u_{\alpha}+\frac{\epsilon n D \tau}{c^{2} b \rho} e_{i \alpha} F_{1 \alpha}\right. \\
+\frac{D(D+2)}{2 c^{4} b}\left(e_{i \alpha} e_{i \beta} u_{\alpha} u_{\beta}+2 \epsilon n \frac{\tau}{\rho} e_{i \alpha} e_{i \beta} F_{1 \alpha} u_{\beta}+\epsilon^{2} \frac{n^{2} \tau^{2}}{\rho^{2}} e_{i \alpha} e_{i \beta} F_{1 \alpha} F_{1 \beta}\right) \\
\left.\quad-\frac{D}{2 c^{2} b}\left(u^{2}+2 \epsilon n \frac{\tau}{\rho} u_{\alpha} F_{1 \alpha}+\epsilon^{2} n^{2} \frac{\tau^{2}}{\rho^{2}} F_{1}^{2}\right)\right], \quad i=1,2, \ldots, b \\
\rho\left[d_{0}-\frac{\epsilon l \phi_{1} D}{c^{2}}-\frac{u^{2}}{c^{2}}-\frac{2 \epsilon n \tau}{c^{2} \rho} u_{\alpha} F_{1 \alpha}-\frac{\epsilon^{2} n^{2} \tau^{2}}{c^{2} \rho^{2}} F_{1}^{2}\right], \quad i=0 .
\end{array}\right.
$$

From this we get $\bar{f}_{i}^{(0)}=E(\rho, \boldsymbol{u})$,

$$
\bar{f}_{i}^{(1)}=\left\{\begin{array}{l}
\rho\left[\frac{l \phi_{1} D}{b c^{2}}+\frac{n D \tau}{c^{2} b \rho} e_{i \alpha} F_{1 \alpha}+\frac{n D(D+2)}{c^{4} b} \frac{\tau}{\rho} e_{i \alpha} e_{i \beta} F_{1 \alpha} u_{\beta}-\frac{n D \tau}{c^{2} b \rho} u_{\alpha} F_{1 \alpha}\right], \quad i=1, b \\
\rho\left[-\frac{\phi_{1} D}{c^{2}}-\frac{2 n \tau}{c^{2} \rho} u_{\alpha} F_{1 \alpha}\right], \quad i=0
\end{array}\right.
$$

and

$$
\bar{f}_{i}^{(2)}=\left\{\begin{array}{l}
\rho\left[\frac{n^{2} D(D+2)}{2 c^{4} b} \frac{\tau^{2}}{\rho^{2}} e_{i \alpha} e_{i \beta} F_{1 \alpha} F_{1 \beta}-\frac{n^{2} D \tau^{2}}{2 c^{2} b^{2} \rho^{2}} F_{1}^{2}\right], \quad i=1,2, \ldots, b \\
\rho\left[\frac{-n^{2} \tau^{2}}{c^{2} \rho^{2}} F_{1}^{2}\right], \quad i=0,
\end{array}\right.
$$

from which we find

$$
\begin{gathered}
\sum_{i} \bar{f}_{i}^{(1)}=0, \sum_{i} \bar{f}_{i}^{(2)}=0, \quad \sum_{i} \bar{f}_{i}^{(1)} e_{i \alpha}=n \tau F_{1 \alpha}, \\
\sum_{i} \bar{f}_{i}^{(2)} e_{i \alpha}=0,
\end{gathered}
$$

and

$$
\sum_{i} \bar{f}_{i}^{(1)} e_{i \alpha} e_{i \beta}=n \tau\left(F_{1 \alpha} u_{\beta}+F_{1 \beta} u_{\alpha}\right)+l \rho \phi_{1} \delta_{\alpha \beta} .
$$

Now, the expansion of the Boltzmann equation up to second order in $\epsilon$ is 


$$
\begin{aligned}
& \epsilon\left(\partial_{1 t} f_{i}^{(0)}+e_{i \alpha} \partial_{1 \alpha} f_{i}^{(0)}\right)+\epsilon^{2}\left(\partial_{2 t} f_{i}^{(0)}+\partial_{1 t} f_{i}^{(1)}+e_{i \alpha} \partial_{1 \alpha} f_{i}^{(1)}\right. \\
& +\frac{1}{2} e_{i \alpha} e_{i \beta} \partial_{1 \alpha} \partial_{1 \beta} f_{i}^{(0)}+\frac{1}{2} e_{i \alpha} \partial_{1 \alpha} \partial_{1 t} f_{i}^{(0)} \\
& \left.\quad+\frac{1}{2} e_{i \alpha} \partial_{1 \alpha} \partial_{1 t} f_{i}^{(0)}+\frac{1}{2} \partial_{1 t} \partial_{1 t} f_{i}^{(0)}\right) \\
& =-\frac{1}{\tau}\left(f_{i}^{(0)}+\epsilon f_{i}^{(1)}+\epsilon^{2} f_{i}^{(2)}-\bar{f}_{i}^{(0)}-\epsilon \bar{f}_{i}^{(1)}-\epsilon^{2} \bar{f}_{i}^{(2)}\right) \\
& +\epsilon \frac{m D}{b c^{2}} F_{1 \alpha} e_{i \alpha} .
\end{aligned}
$$

This gives $O\left(\epsilon^{0}\right)$,

$$
f_{i}^{(0)}=\bar{f}_{i}^{(0)},
$$

$O(\epsilon)$,

$$
\partial_{1 t} f_{i}^{(0)}+e_{i \alpha} \partial_{1 \alpha} f_{i}^{(0)}=-\frac{1}{\tau}\left(f_{i}^{(1)}-\bar{f}_{i}^{(1)}\right)+\frac{m D}{b c^{2}} F_{1 \alpha} e_{i \alpha},
$$

and $O\left(\epsilon^{2}\right)$,

$$
\begin{aligned}
\partial_{2 t} f_{i}^{(0)} & +\partial_{1 t} f_{i}^{(1)}+e_{i \alpha} \partial_{1 \alpha} f_{i}^{(1)}+\frac{1}{2} e_{i \alpha} e_{i \beta} \partial_{1 \alpha} \partial_{1 \beta} f_{i}^{(0)} \\
& +\frac{1}{2} e_{i \alpha} \partial_{1 \alpha} \partial_{1 t} f_{i}^{(0)}+\frac{1}{2} e_{i \alpha} \partial_{1 \alpha} \partial_{1 t} f_{i}^{(0)}+\frac{1}{2} \partial_{1 t} \partial_{1 t} f_{i}^{(0)} \\
= & -\frac{1}{\tau}\left(f_{i}^{(2)}-\bar{f}_{i}^{(2)}\right) .
\end{aligned}
$$

We can now sum Eqs. (A10) and (A11) and their product with $\boldsymbol{e}_{i}$ where $\Sigma_{i} f_{i}^{(1)}=\Sigma_{i} f_{i}^{(1)} e_{i \alpha}=\Sigma_{i} f_{i}^{(2)}=\Sigma_{i} f_{i}^{(2)} e_{i \alpha}=0$. Summing Eq. (A10) gives

$$
\partial_{1 t} \rho+\partial_{1 \alpha} \rho u_{\alpha}=0,
$$

while multiplying by $e_{i \beta}$ before summing gives

$$
\partial_{1 t} \rho u_{\beta}+\partial_{1 \alpha} \rho u_{\alpha} u_{\beta}=-\frac{\left(1-d_{0}\right)}{D} c^{2} \partial_{1 \beta} \rho+(n+m) F_{1 \beta} .
$$

Summing Eq. (A11) gives

$$
\partial_{2 t} \rho+\frac{1}{2} \partial_{1 \alpha}(n+m) F_{1 \alpha}=0,
$$

where we have used Eqs. (A12) and (A13). Multiplying by $e_{i \gamma}$ before summing gives

$$
\begin{gathered}
\partial_{2 t} \rho u_{\gamma}+\frac{1}{2} \partial_{1 t}(n+m) F_{1 \gamma}+\partial_{1 \alpha} n \tau\left(F_{1 \gamma} u_{\alpha}+F_{1 \alpha} u_{\gamma}\right) \\
+\partial_{1 \gamma} l \rho \phi_{1}=\nu \partial_{1 \alpha} \partial_{1 \alpha} \rho u_{\gamma}+\zeta \partial_{1 \gamma} \partial_{1 \alpha} \rho u_{\alpha},
\end{gathered}
$$

where we have used

$$
\begin{aligned}
\sum_{i} e_{i \alpha} e_{i \beta} f_{i}^{(1)}= & n \tau\left(F_{1 \alpha} u_{\beta}+F_{1 \beta} u_{\alpha}\right) \\
& -\tau \partial_{1 t}\left(\frac{\rho\left(1-d_{0}\right) c^{2}}{D} \delta_{\alpha \beta}+\rho u_{\alpha} u_{\beta}\right) \\
& -\tau \partial_{1 \gamma} \frac{\rho c^{2}}{(D+2)}\left(u_{\alpha} \delta_{\beta \gamma}+u_{\beta} \delta_{\alpha \gamma}+u_{\gamma} \delta_{\alpha \beta}\right) \\
& +\rho \phi_{1} l \delta_{\alpha \beta},
\end{aligned}
$$

which is found from Eq. (A10). Combining Eqs. (A12) and (A14) gives

$$
\partial_{t} \rho+\partial_{\alpha}\left[\rho u_{\alpha}+\frac{1}{2}(n+m) F_{\alpha}\right]=0,
$$

while combining Eqs. (A13) and (A15) gives

$$
\begin{aligned}
\partial_{t}\left[\rho u_{\alpha}+\frac{1}{2}(n+m) F_{1 \alpha}\right]+\partial_{\beta}\left[\rho u_{\alpha} u_{\beta}+\tau n\left(u_{\alpha} F_{\beta}+u_{\beta} F_{\alpha}\right)\right] \\
=-\partial_{\alpha}\left(\frac{\rho\left(1-d_{0}\right) c^{2}}{D}\right)+\nu \partial_{\beta} \partial_{\beta} \rho u_{\alpha}+\zeta \partial_{1 \alpha} \partial_{1 \beta} \rho u_{\beta} \\
\quad+(n+m) F_{\alpha}-l \partial_{\alpha} \rho \phi .
\end{aligned}
$$

\section{APPENDIX B: ANALYTICAL RESULTS}

We now consider exact analytical solutions $[25,26,30]$ for methods (1)-(4) applied to a two-dimensional hexagonal grid $(D=2, b=6)$. In doing this we assume a steady solution invariant in the $x$ direction. This steady state implies

$$
f_{0}(z)=\bar{f}_{0}(z), f_{2}(z)=\bar{f}_{2}(z), f_{5}(z)=\bar{f}_{5}(z), \quad \forall z,
$$

where $\boldsymbol{e}_{i}=\sin (\pi i / 3-\pi / 6) \hat{\boldsymbol{e}}_{x}+\cos (\pi i / 3-\pi / 6) \hat{\boldsymbol{e}}_{z}, \hat{\boldsymbol{e}}_{x}$ and $\hat{\boldsymbol{e}}_{z}$ are unit vectors in the horizontal and vertical directions, respectively, and gravity acts in the $z$ direction. We are solving the lattice Boltzmann equation,

$$
f_{i}\left(\boldsymbol{r}+\boldsymbol{e}_{i}, t+1\right)-f_{i}(\boldsymbol{r}, t)=-\frac{1}{\tau}\left(f_{i}-\bar{f}_{i}\right)+m \frac{D}{b c^{2}} F_{\alpha} e_{i \alpha},
$$

where

$$
\bar{f}_{i}=E_{i}^{\prime}(\rho, \boldsymbol{u}+n \boldsymbol{w})
$$

and $\boldsymbol{w}=\tau \boldsymbol{F} / \rho$. This represents method (1) for $l=1, m=n$ $=0$, method (2) for $m=1, l=n=0$, method (3) for $n=1, l$ $=m=0$, and method (4) for $l=0, m=(2 \tau-1) /(2 \tau), n$ $=1 /(2 \tau)$. We also define $\boldsymbol{v}$ according to Eq. (16). Now $\sum_{i} f_{i}=\sum_{i} \bar{f}_{i}$ and $\sum_{i} f_{i} e_{i x}=\Sigma_{i} \bar{f}_{i} e_{i x}$, while $\sum_{i} f_{i} e_{i z}=\sum_{i} \overline{f_{i}} e_{i z}$ $+n \tau F_{z}$. This gives

$$
\begin{aligned}
& f_{1}(z)+f_{3}(z)+f_{4}(z)+f_{6}(z)=\bar{f}_{1}(z)+\bar{f}_{3}(z)+\bar{f}_{4}(z)+\bar{f}_{6}(z), \\
& f_{1}(z)+f_{3}(z)-f_{4}(z)-f_{6}(z)=\bar{f}_{1}(z)+\bar{f}_{3}(z)-\bar{f}_{4}(z)-\bar{f}_{6}(z),
\end{aligned}
$$




$$
\begin{aligned}
f_{1}(z)-f_{3}(z)-f_{4}(z)+f_{6}(z)= & \bar{f}_{1}(z)-\bar{f}_{3}(z)-\bar{f}_{4}(z)+\bar{f}_{6}(z) \\
& -\frac{2}{\sqrt{3}} \tau F_{z}(z) .
\end{aligned}
$$

Assuming that $u_{x}(z)=0, \forall z$ these have solution

$f_{3}(z)=f_{4}(z), f_{1}(z)=f_{6}(z), \bar{f}_{3}(z)=\bar{f}_{4}(z), \bar{f}_{1}(z)=\bar{f}_{6}(z)$,

$$
f_{1}(z)=\bar{f}_{1}(z)-\frac{n \tau F_{z}(z)}{2 \sqrt{3}}, \text { and } f_{3}(z)=\bar{f}_{3}(z)+\frac{n \tau F_{z}(z)}{2 \sqrt{3}} .
$$

Now the Boltzmann equation for $i=1$ and $i=3$ combined with Eq. (B5) gives

$$
\begin{gathered}
\bar{f}_{1}(z+\sqrt{3} / 2)-\frac{n \tau F_{z}(z+\sqrt{3} / 2)}{2 \sqrt{3}} \\
=\bar{f}_{1}(z)-\frac{n \tau F_{z}(z)}{2 \sqrt{3}} \\
+(n+m) \frac{F_{z}(z)}{2 \sqrt{3}}
\end{gathered}
$$

and

$$
\begin{aligned}
& \bar{f}_{3}(z-\sqrt{3} / 2)+\frac{n \tau F_{z}(z-\sqrt{3} / 2)}{2 \sqrt{3}} \\
& =\bar{f}_{3}(z)+\frac{n \tau F_{z}(z)}{2 \sqrt{3}}-(n+m) \frac{F_{z}(z)}{2 \sqrt{3}} .
\end{aligned}
$$

Replacing $\bar{f}_{i}$ with $E_{i}^{\prime}(\rho, \boldsymbol{u}+n \boldsymbol{w})$ in Eqs. (B6) and (B7), noting that $c=1$ in lattice units and replacing $z$ with $z+\sqrt{3} / 2$ in Eq. (B7), gives

$$
\begin{aligned}
\rho(z+ & \sqrt{3} / 2)\left\{\left(1-d_{0}\right)+2 l \phi(z+\sqrt{3} / 2)+\sqrt{3} u_{z}(z+\sqrt{3} / 2)\right. \\
& \left.+2\left[v_{z}(z+\sqrt{3} / 2)+(n \tau-1 / 2) \frac{F_{z}(z+\sqrt{3} / 2)}{\rho(z+\sqrt{3} / 2)}\right]^{2}\right\} \\
= & \rho(z)\left\{\left(1-d_{0}\right)+2 l \phi(z)+\sqrt{3} u_{z}(z)\right. \\
& \left.+2\left[v_{z}(z)+(n \tau-1 / 2) \frac{F_{z}(z)}{\rho(z)}\right]^{2}\right\} \\
& +\sqrt{3}(n+m) F_{z}(z)
\end{aligned}
$$

$$
\begin{aligned}
\rho(z+ & \sqrt{3} / 2)\left\{\left(1-d_{0}\right)+2 l \phi(z+\sqrt{3} / 2)-\sqrt{3} u_{z}(z+\sqrt{3} / 2)\right. \\
& \left.+2\left[v_{z}(z+\sqrt{3} / 2)+(n \tau-1 / 2) \frac{F_{z}(z+\sqrt{3} / 2)}{\rho(z+\sqrt{3} / 2)}\right]^{2}\right\} \\
& -(n+m) \sqrt{3} F_{z}(z+\sqrt{3} / 2) \\
= & \rho(z)\left\{\left(1-d_{0}\right)+2 l \phi(z)-\sqrt{3} u_{z}(z)\right. \\
& \left.+2\left[v_{z}(z)+(n \tau-1 / 2) \frac{F_{z}(z)}{\rho(z)}\right]^{2}\right\} .
\end{aligned}
$$

Subtracting Eq. (B9) from Eq. (B8) gives

$$
\begin{aligned}
& 2 \rho(z+\sqrt{3} / 2) u_{z}(z+\sqrt{3} / 2)+(n+m) F_{z}(z+\sqrt{3} / 2) \\
& \quad=2 \rho(z) u_{z}(z)+(n+m) F_{z}(z),
\end{aligned}
$$

while adding the equations gives

$$
\begin{aligned}
\rho(z+ & \sqrt{3} / 2)\left\{2\left(1-d_{0}\right)+4\left[v_{z}(z+\sqrt{3} / 2)+(n \tau-1 / 2)\right.\right. \\
& \left.\left.\times \frac{F_{z}(z+\sqrt{3} / 2)}{\rho(z+\sqrt{3} / 2)}\right]^{2}\right\}+4 l \rho(z+\sqrt{3} / 2) \phi(z+\sqrt{3} / 2)-(m \\
& +n) \sqrt{3} F_{z}(z+\sqrt{3} / 2) \\
= & \rho(z)\left\{2\left(1-d_{0}\right)+4\left[v_{z}(z)+(n \tau-1 / 2) \frac{F_{z}(z)}{\rho(z)}\right]^{2}\right. \\
& +4 l \phi(z)\}+(m+n) \sqrt{3} f_{z}(z) .
\end{aligned}
$$

Now consider a bounce-back boundary condition between the last fluid site at $z=z_{0}$ and an obstacle at $z=\left(z_{0}-\sqrt{3} / 2\right)$. This can be expressed as

$$
f_{1}\left(z_{0}\right)=f_{4}\left(z_{0}-\sqrt{3} / 2\right) .
$$

Using Eqs. (B5) and (B7) this can be written as

$$
\bar{f}_{1}\left(z_{0}\right)-\frac{n \tau F_{z}\left(z_{0}\right)}{2 \sqrt{3}}=\bar{f}_{3}\left(z_{0}\right)+\frac{n \tau F_{z}\left(z_{0}\right)}{2 \sqrt{3}}-(n+m) \frac{F_{z}\left(z_{0}\right)}{2 \sqrt{3}} .
$$

Since $\bar{f}_{i}=E_{i}^{\prime}(\rho, \boldsymbol{u}+n \boldsymbol{w})$ this gives the boundary condition

$$
2 \rho\left(z_{0}\right) u_{z}\left(z_{0}\right)+(n+m) F_{z}\left(z_{0}\right)=0 .
$$

Consider first methods (2), (3), and (4), which have $l$ $=0$ and $m+n=1$. In these cases the boundary condition Eq. (B14) becomes

$$
2 \rho\left(z_{0}\right) v_{z}\left(z_{0}\right)=0 \text { or } \rho_{0} v_{z}\left(z_{0}\right)=0,
$$

where $\rho_{0}=\rho\left(z_{0}\right)$. Combining Eq. (B10) with this boundary condition gives

$$
\rho(z) v_{z}(z)=\rho_{0} v_{z}\left(z_{0}\right)=0,
$$


which shows that the bounce-back condition imposes a confined fluid under gravity to be at rest only if the velocity is taken to be $\boldsymbol{v}$ as is the case for methods (2), (3), and (4).

Setting $v_{z}=0$ in Eq. (B11) to find the long-term behavior of the fluid and $F_{z}(z)=-\rho(z) g$ gives

$$
\begin{aligned}
& \rho(z+\sqrt{3} / 2) \\
& \quad=\rho(z)\left[\frac{1-\sqrt{3} g / 2\left(1-d_{0}\right)+2(n \tau-1 / 2)^{2} g^{2} /\left(1-d_{0}\right)}{1+\sqrt{3} g / 2\left(1-d_{0}\right)+2(n \tau-1 / 2)^{2} g^{2} /\left(1-d_{0}\right)}\right]
\end{aligned}
$$

or

$$
\begin{aligned}
& \rho(z) \\
& \quad=\rho_{0}\left[\frac{1-\sqrt{3} g / 2\left(1-d_{0}\right)+2(n \tau-1 / 2)^{2} g^{2} /\left(1-d_{0}\right)}{1+\sqrt{3} g / 2\left(1-d_{0}\right)+2(n \tau-1 / 2)^{2} g^{2} /\left(1-d_{0}\right)}\right]^{2 z / \sqrt{3}} .
\end{aligned}
$$

When $\tau=1$ the density gradient produced by both methods (2) and (3) will be the same, although different from the gradient produced by method (4), despite it being a composite of the two methods. If $\tau \neq 1$ the density gradient will differ between the different methods, for method (3) this will depend on $\tau$ and for methods (2) and (4) the gradient is independent of $\tau$, however these differences will in general be small. In the incompressible limit, $g z \ll c_{s}^{2}$, we expect $\sqrt{3} g / 2 \gg(n \tau-1 / 2)^{2} g^{2}$ for the values of $\tau$ typically used in lattice Boltzmann simulations; for method (4) this is always true since $n \tau-1 / 2=0$. In this incompressible limit

$$
\rho(z) \simeq \rho_{0}\left(1-\frac{2 g z}{1-d_{0}}\right)
$$

for each method. It is worth noting that in the compressible limit the exponential law, $\rho(z)=\rho_{0} \exp \left(g^{\prime} z / c_{s}^{2}\right)$, is obtained in terms of a rescaled gravity

$$
g^{\prime}=\frac{2 c_{s}^{2}}{\sqrt{3}} \ln \left[\frac{1-\sqrt{3} g / 2\left(1-d_{0}\right)+2(n \tau-1 / 2)^{2} g^{2} /\left(1-d_{0}\right)}{1+\sqrt{3} g / 2\left(1-d_{0}\right)+2(n \tau-1 / 2)^{2} g^{2} /\left(1-d_{0}\right)}\right] \text {. }
$$

For method (1) we have $l=1$ and $m=n=0$. In this case a similar argument leads to

$$
\rho(z) u_{z}(z)=\rho_{0} u_{z}\left(z_{0}\right)=0
$$

implying that the fluid velocity should be taken as $\boldsymbol{u}$, and

$$
\begin{aligned}
& \rho(z+\sqrt{3} / 2)\left[\left(1-d_{0}\right)+2 u_{z}(z+\sqrt{3} / 2)+2 \phi(z+\sqrt{3} / 2)\right] \\
& \quad=\rho(z)\left[\left(1-d_{0}\right)+2 u_{z}(z)+2 \phi(z)\right] .
\end{aligned}
$$

Since method (1) can only be applied if there is a negligible change in the fluid density it is not sensible to consider a density gradient since this is negligibly small. Here we consider the density variation in this limit to enable a comparison to be made with the other methods. In this limit we can write

$$
\begin{aligned}
& 2 \rho(z+\sqrt{3} / 2) \phi(z+\sqrt{3} / 2)-2 \rho \phi(z) \\
& \quad \simeq 2 \rho(z)[\phi(z+\sqrt{3} / 2)-\phi(z)] \\
& \quad \simeq \sqrt{3} \rho(z) \nabla \phi(z) .
\end{aligned}
$$

Or, to the same approximation

$$
2 \rho(z+\sqrt{3} / 2) \phi(z+\sqrt{3} / 2)-2 \rho \phi(z) \simeq \sqrt{3} \rho(z) \nabla \phi(z) .
$$

This approximation allows Eq. (B11) to be expressed as

$$
\rho(z+\sqrt{3} / 2)\left[2\left(1-d_{0}\right)+\sqrt{3} g\right] \simeq \rho(z)\left[2\left(1-d_{0}\right)-\sqrt{3} g\right]
$$

giving

$$
\rho(z) \simeq \rho_{0}\left(1-\frac{2 g z}{1-d_{0}}\right)
$$

as was found for the other methods.
[1] G. R. McNamara and G. Zanetti, Phys. Rev. Lett. 61, 2332 (1988).

[2] F. J. Higuera, S. Succi, and R. Benzi, Europhys. Lett. 9, 345 (1989).

[3] H. Chen, S. Chen, and H. Matthaeus, Phys. Rev. A 45, 5339 (1992).

[4] Y. H. Qian, D. d' Humières, and P. Lallemand, Europhys. Lett. 17, 479 (1992).

[5] U. Frisch, B. Hasslacher, and Y. Pomeau, Phys. Rev. Lett. 56, 1505 (1986).

[6] S. Chen, H. Chen, D. Martinez, and W. Matthaues, Phys. Rev. Lett. 67, 3776 (1991).

[7] S. Chen, Z. Wang, X. Shan, and G. D. Doolen, J. Stat. Phys. 68, 379 (1992).

[8] S. Succi, R. Benzi, and F. Higuera, Physica D 47, 219 (1991).
[9] A. J. C. Ladd, J. Fluid Mech. 271, 311 (1994).

[10] E. G. Flekkøy, Phys. Rev. E 47, 4247 (1993).

[11] X. Shan and H. Chen, Phys. Rev. E 47, 1815 (1993).

[12] M. R. Swift, E. Orlandini, W. R. Osborn, and J. M. Yeomans, Phys. Rev. E 54, 5041 (1996).

[13] X. He, L.-S. Luo, and M. Dembo, J. Comput. Phys. 129, 357 (1996).

[14] J. Lighthill, Waves In Fluids (Cambridge University Press, Cambridge, England, 1978).

[15] J. S. Turner, Buoyancy Effects in Fluids (Cambridge University Press, Cambridge, England, 1973).

[16] J. M. Buick, C. A. Greated, and W. J. Easson, Phys. Fluids 9, 2585 (1997).

[17] U. Frisch, D. d' Humières, B. Hasslacher, P. Lallemand, and Y. Pomeau, Complex Syst. 1, 649 (1987). 
[18] S. Wolfram, J. Stat. Phys. 45, 471 (1986).

[19] S. Chapman and T. G. Cowling, The Mathematical Theory of Non-Uniform Gases (Cambridge University Press, Cambridge, 1970).

[20] C. Cercignani, Theory and Application of the Boltzmann Equation (Scottish Academic Press, Edinburgh, U.K., 1975).

[21] P. L. Bhatnagar, E. P. Gross, and M. Krook, Phys. Rev. 94, 511 (1954).

[22] R. D. Kingdon, Technical Report No. AEA-InTec-1298, AEA Technology, 1993, (unpublished).

[23] N. S. Martys and H. Chen, Phys. Rev. E 53, 743 (1996).
[24] X. Shan and G. Doolen, J. Stat. Phys. 81, 379 (1995).

[25] I. Ginzbourg and P. M. Alder, J. Phys. II 4, 191 (1994).

[26] X. He, Q. Zou, L.-S. Luo, and M. Dembo, J. Stat. Phys. 87, 115 (1997).

[27] D. R. Noble, S. Chen, J. G. Georgiadis, and R. O. Buckius, Phys. Fluids 7, 203 (1995).

[28] J. D. Sterling and S. Chen, J. Comput. Phys. 123, 196 (1996).

[29] E. Orlandini, M. R. Swift, and J. M. Yeomans, Europhys. Lett. 32, 463 (1995).

[30] Q. Zou, S. Hou, and G. D. Doolen, J. Stat. Phys. 81, 319 (1995). 